\title{
Virtual Force Concept in Steering Mobile Manipulators with Skid-Steering Platform Moving in Unknown Environment
}

\author{
Alicja Mazur • Mateusz Cholewiński
}

Received: 15 January 2013 / Accepted: 30 May 2013 / Published online: 27 September 2013

(C) The Author(s) 2013. This article is published with open access at SpringerLink.com

\begin{abstract}
Underactuated control system, namely mobile manipulator mounted on skid-steering platform has been considered in the paper. As a control scheme the concept of virtual force is presented and discussed. In such concept, full rank of input matrix, describing relationship between controlled variables and number of actuators, has been assumed. The signal of the assumed additional actuator is equal to zero equivalently (because this force is "virtual" and it does not exist in practice) but in this way the invertibility of input matrix can be achieved. The equation of the implicit function generates the reference signals needed in control algorithm. Mathematical proof of proper action of such control law has been presented using Lyapunov-like function. Efficiency and robustness of control scheme on parametric uncertainty of terrain, which the mobile manipulator is moving on, have been tested in numerous simulations.
\end{abstract}

Keywords Mobile robot - Nonholonomic constraints $\cdot$ Parametric uncertainty $\cdot$ Robust control

\section{Introduction}

Mobile manipulator, which is the subject of consideration, consists of a mobile platform and an onboard rigid manipulating arm. Such robotic object can execute more complicated tasks than its components. Manipulating arm is fully controlled while mobile platform equipped with more than one axis of fixed wheels is an underactuated system.

Wheeled mobile platforms constitute important group of robotic objects. They can be treated as independent robots or as an transportation part of composed robotic assemble, for instance mobile manipulators. Depending on wheels' kind and the way in which they are fixed to the cart, motion of wheeled mobile platforms can be realized with or without slippage phenomenon. If no slippage effect between wheels and surface occurs, then exists some equation describing forbidden directions of realized velocities of the system. Such a relationship is called nonholonomic constraint in platform's motion.

Special kind of wheeled mobile platforms are platforms with more than one axis equipped with fixed wheels, see Fig. 1. These platforms are called

e-mail: mateusz.cholewinski@pwr.wroc.pl 


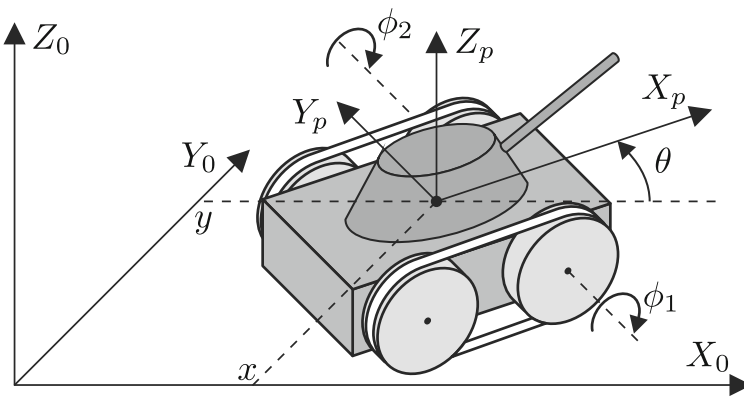

Fig. 1 Schematic of mobile manipulator with SSMP platform - tank

skid-steering mobile platforms (SSMP), due to skidding effect observed in theirs behavior [4] for details.

Research activities in the field of modeling and developing of control algorithms for SSMP platforms have been continued from the last 90's of XX century. First attempt to solve this problem can be found in the work [2], in which some assumption about instantaneous center of rotation (ICR) during the platform's motion has been done. Authors made assumption that there exists some non-integrable relationship between angular velocity (orientation's changes) and lateral slipping of wheels. Such equation, although derived from slipping effect, could play a role of special nonholonomic constraint. Similar approach to this presented by Caracciolo et al. can be found in [4] and in other papers.

Another approach to the problem of modeling and control of SSMP platform has occurred recently, e.g. in $[8,9]$. The authors have observed that the assumption introduced by Caracciolo et al. is not always fulfilled in practice and it should be extended on accelerations in platform's motion. They have treated SSMP platform as an underactuated system on dynamic level with non-stationary kinematics (non-stationary velocity constraint). Both concepts, i.e. with stationary or non-stationary constraint on velocity, need system of motion planning, solving nonholonomic constraints in any time. Such system is sophisticated and complicated.

It is possible to describe SSMP platform in another way. Such idea, known as a singular perturbation approach, can be found in the paper [3].
The authors have introduced new variable for slippage and have reformulated mathematical equations including such variable in dynamical model. This method is not very often used in practical applications because it is very complex and it tries to strictly evaluate slippage phenomenon which can be unpredictable.

In this paper a new concept of control problem of mobile manipulator's with SSMP platform has been presented. Instead of motion planner, mobile manipulator with SSMP platform can be considered as an underactuated system with rectangular input matrix. Problem of inverting of such matrix (standing near control signals) can be solved with the idea of so-called "virtual force".

The paper is organized in the following way. In Section 2 concept of proposed approach to the control nonholonomic mobile manipulators is presented. Section 3 illustrates theoretical design of mathematical model of considered objects. In Section 4 the control problem is formulated. In Section 5 a new control algorithm is designed and its proper action is proved. Section 6 contains the simulation results. Section 7 presents some conclusions.

\section{Concept of Virtual Force}

As mentioned earlier in the introduction, mobile manipulator with SSMP platform is underactuated system: number of controlled system variables is bigger than number of control inputs. It implies the rectangular form of input matrix $B$ and, consequently, a problem with its inverse and control design. In this paper a new approach to control of SSMP platform will be presented. In this method it is being assumed that controlled system has full number of input signals and therefore input matrix is invertible. These control inputs, which do not exist in reality, will be called "virtual forces". Since virtual forces are not real (they do not exist in practice), in further considerations it will be assumed that they are always equal to zero equivalently, i.e.

$u_{i, \text { virtual }} \equiv 0, \quad i=1, m-n$.

Equation 1 defining virtual forces are implicit functions including state variables, regulation 
parameters and desired trajectories. From Eq. 1 it is possible to obtain missing reference signals, needed for different control algorithms.

Similar approach to the shortage in control inputs in underactuated systems can be found in literature, e.g. for nonholonomic inverted pendulum mounted on unicycle [6], in control algorithms using extended Jacobi matrix for redundant manipulators [1] or in endogenous configuration space approach for nonholonomic mobile manipulators [10]. However, concept of virtual force for SSMP platform is introduced and considered in this paper the first time.

\section{Mathematical Models in Different Coordinates for Mobile Manipulator with SSMP Platform}

Let's consider rigid manipulator mounted on the SSMP platform with two axes of fixed wheels, presented in Fig. 1. State of such object can be described by vector of platform generalized variables $q_{m}$ and manipulator variables $q_{r}$, i.e. $q=$ $\left(q_{m}, q_{r}\right)^{T}$

$$
q_{m}=\left(\begin{array}{lllll}
x & y & \theta & \phi_{1} & \phi_{2}
\end{array}\right)^{T}, \quad q_{r}=\left(\begin{array}{lll}
q_{1} & q_{2} \ldots q_{p}
\end{array}\right)^{T}
$$

where $(x, y)$ are cartesian coordinates of mass center defined relative to global frame $X_{0} Y_{0}, \theta$ is an orientation of SSMP platform, $\phi_{i}$ is an angle of rotation of wheels located on the right or the left side, whereas $q_{r}$ is a vector of manipulator joint variables. For selected generalized coordinates of SSMP platform there exists some relationship [4]

$$
\left(\begin{array}{c}
\dot{x} \\
\dot{y} \\
\dot{\theta}
\end{array}\right)=\operatorname{Rot}(z, \theta)\left(\begin{array}{c}
v_{x} \\
v_{y} \\
\omega
\end{array}\right) .
$$

Symbols $v_{x}$ and $v_{y}$ mean elements of onward velocity relative to local axes $X_{p}$ and $Y_{p}$ while $\omega$ is angular velocity of the SSMP platform.

In this paper the only assumption about wheels motion without longitudinal slip has been taken. Moreover, we assume that velocity of considered mobile manipulator is rather small, not bigger that $10\left[\frac{\mathrm{km}}{\mathrm{h}}\right]$.
All wheels of SSMP platform are identical, therefore constraints on lack of longitudinal slip can be expressed as follows

$\dot{\phi}_{1} r=v_{x}+c \omega \quad$ right side of the platform,

$\dot{\phi}_{2} r=v_{x}-c \omega \quad$ left side of the platform,

where $r$ is a radius of wheel and $c$ is a half of platform width. Equations 2-3 can be expressed in so-called Pfaffian form in the following way

$$
A\left(q_{m}\right) \dot{q}_{m}=\left[\begin{array}{cccc}
\cos \theta \sin \theta & -c & -r & 0 \\
\cos \theta \sin \theta & c & 0 & -r
\end{array}\right]\left(\begin{array}{c}
\dot{x} \\
\dot{y} \\
\dot{\theta} \\
\dot{\phi}_{1} \\
\dot{\phi}_{2}
\end{array}\right)=0 .
$$

\subsection{Model in Generalized Coordinates}

For nonholonomic, skid-steering vehicles without longitudinal slippage, dynamics could be derived from d'Alembert principle as

$$
\begin{aligned}
M(q) \ddot{q}+ & C(q, \dot{q}) \dot{q}+D(q)+F(q, \dot{q})=B(q) u \\
& +A^{T}\left(q_{m}\right) \lambda,
\end{aligned}
$$

or more in detail

$$
\begin{gathered}
{\left[\begin{array}{ll}
M_{11} & M_{12} \\
M_{21} & M_{22}
\end{array}\right]\left(\begin{array}{l}
\ddot{q}_{m} \\
\ddot{q}_{r}
\end{array}\right)+\left[\begin{array}{ll}
C_{11} & C_{12} \\
C_{21} & C_{22}
\end{array}\right]\left(\begin{array}{c}
\dot{q}_{m} \\
\dot{q}_{r}
\end{array}\right)+\left(\begin{array}{c}
0 \\
D
\end{array}\right)} \\
+\left(\begin{array}{c}
F \\
0
\end{array}\right)=\left[\begin{array}{ll}
B & 0 \\
0 & I
\end{array}\right]\left(\begin{array}{c}
u_{m} \\
u_{r}
\end{array}\right)+\left(\begin{array}{c}
A^{T} \lambda \\
0
\end{array}\right)
\end{gathered}
$$

Symbol $M(q)$ denotes inertia matrix of mobile manipulator, matrix $C(q, \dot{q})$ describes Coriolis and centripetal forces, vector $D(q)$ is a vector of gravity forces (only for manipulating arm because platform moves on horizontal surface), while vector $F(q, \dot{q})$ is a vector of non-potential forces, mostly reaction forces from terrain and friction forces. Torques $B(q) u$ represent control inputs (actuators) whereas $A^{T} \lambda$ are forces coming from nonholonomic constraints. Matrix $B(q)$, so-called 
input matrix, describes relationship between input signals $u$ and torques coming from motor shafts

$$
B \cdot u_{m}=\left[\begin{array}{ccc}
0 & 0 & -\sin \theta \\
0 & 0 & \cos \theta \\
0 & 0 & 0 \\
0 & 1 & 0 \\
1 & 0 & 0
\end{array}\right]\left(\begin{array}{l}
u_{1} \\
u_{2} \\
u_{v}
\end{array}\right)
$$

Symbols $u_{1}$ and $u_{2}$ describe control signal associated with coupled wheels on the right and left side of the SSMP platform. Third column of matrix $B(q)$ is responsible for hypothetical virtual force $u_{v}$, influenced on platform orientation.

Equations 4 and 5 constitute complete model of special nonholonomic system, namely mobile manipulator with SSMP platform, expressed in generalized coordinates.

\subsection{Model in Auxiliary Coordinates}

Mobile manipulator with SSMP platform is a control system with nonholonomic constraints. These constraints, called kinematics of nonholonomic system, can be transformed to auxiliary coordinates

- kinematics - driftless control system

$\dot{q}_{m}=G(q) \eta=\left[\begin{array}{rrr}\cos \theta & \cos \theta & -\sin \theta \\ \sin \theta & \sin \theta & \cos \theta \\ \frac{1}{c} & -\frac{1}{c} & 0 \\ 0 & \frac{2}{r} & 0 \\ \frac{2}{r} & 0 & 0\end{array}\right]\left(\begin{array}{l}\eta_{1} \\ \eta_{2} \\ \eta_{3 v}\end{array}\right)$.

On the other hand, dynamics expressed in auxiliary velocities for nonholonomic subsystem have a form

- dynamics

$M^{*}\left(\begin{array}{c}\dot{\eta} \\ \ddot{q}_{r}\end{array}\right)+C^{*}\left(\begin{array}{c}\eta \\ \dot{q}_{r}\end{array}\right)+\left(\begin{array}{c}0 \\ D\end{array}\right)+\left(\begin{array}{c}F^{*} \\ 0\end{array}\right)=B^{*}\left(\begin{array}{c}u_{m} \\ u_{r}\end{array}\right)$, where:

$$
\begin{aligned}
M^{*} & =\left[\begin{array}{cc}
G^{T} M_{11} G & G^{T} M_{12} \\
M_{21} G & M_{22}
\end{array}\right], \\
C^{*} & =\left[\begin{array}{cc}
G^{T}\left(C_{11} G+M_{11} \dot{G}\right) & G^{T} C_{12} \\
M_{21} \dot{G}+C_{21} G & C_{22}
\end{array}\right], \\
F^{*} & =G^{T} F, \\
B^{*} & =\left[\begin{array}{cc}
G^{T} B & 0 \\
0 & I
\end{array}\right] .
\end{aligned}
$$

\subsection{Model in Linearizing Coordinates}

For SSMP platform, it is possible to choose another coordinates for description, e.g. so-called linearizing outputs

$\xi=\left(\begin{array}{l}\xi_{1} \\ \xi_{2} \\ \xi_{3}\end{array}\right)=\left(\begin{array}{c}x+l \cos \theta \\ y+l \sin \theta \\ \theta\end{array}\right)$.

From [7] it is known that number of linearizing outputs is equal to the difference between dimension of state variables and number of nonholonomic constraints. It means that it is possible to get three linearizing variables for SSMP platform.

First and second element of $\xi$ are coordinates of a point lying in distance $l$ to front of the platform, whereas last element is an orientation of the platform.

Relationship between auxiliary and linearizing coordinates for nonholonomic SSMP platform has a matrix form [7]

$R\left(q_{m}\right) \dot{\xi}=\eta$

with matrix $R\left(q_{m}\right)$

$R\left(q_{m}\right)=\left[\begin{array}{ccc}\frac{\cos \theta}{2} & \frac{\sin \theta}{2} & -\frac{c}{2} \\ \frac{\cos \theta}{2} & \frac{\sin \theta}{2} & \frac{c}{2} \\ -\sin \theta & \cos \theta & -l\end{array}\right]$,

with determinant equal to $-\frac{c}{2}$.

In turn, dynamics of considered mobile manipulator can be expressed in $\left(\xi, q_{r}\right)$ variables in the following way

$J_{\xi}\left(\begin{array}{c}\ddot{\xi} \\ \ddot{q}_{r}\end{array}\right)+C_{\xi}\left(\begin{array}{c}\dot{\xi} \\ \dot{q}_{r}\end{array}\right)+\left(\begin{array}{c}0 \\ D\end{array}\right)+\left(\begin{array}{c}F_{\xi} \\ 0\end{array}\right)=B_{\xi}\left(\begin{array}{c}u_{m} \\ u_{r}\end{array}\right)$, 
where selected elements of model (8) can be calculated as follows

$$
\begin{aligned}
J_{\xi} & =\left[\begin{array}{cc}
R^{T} G^{T} M_{11} G R & R^{T} G^{T} M_{12} \\
M_{21} G R & M_{22}
\end{array}\right], \\
B_{\xi} & =\left[\begin{array}{rr}
R^{T} G^{T} B & 0 \\
0 & I
\end{array}\right], \\
C_{\xi} & =\left[\begin{array}{cc}
R^{T} G^{T}\left(C_{11} G R+M_{11} \dot{G} R+M_{11} G \dot{R}\right) & R^{T} G^{T} C_{12} \\
M_{21} G \dot{R}+M_{21} \dot{G} R+C_{21} G R & C_{22}
\end{array}\right], \\
F_{\xi} & =R^{T} G^{T} F .
\end{aligned}
$$

Equation 8 is a mathematical model of mobile manipulator with SSMP platform expressed in $\left(\xi, q_{r}\right)$ variables.

\section{Control Problem Statement}

In this paper mobile manipulator with SSMP platform is considered. Such robotic object should track desired trajectory without longitudinal slip of its wheels. It implies appearance of nonholonomic constraints (4) in mobile manipulator's motion. By designing of control law we take an assumption that dynamics are expressed in $\left(\xi, q_{r}\right)$ coordinates, i.e. given by Eq. 8. Our goal is to address the following control problem to considered object:

1. Determine control law $u$ in such a way that mobile manipulator with SSMP platform, with fully known dynamics, follows the desired trajectory on unknown terrain without any longitudinal slip of wheels.

2. All virtual forces have to be equal to zero, because they do not exist in reality.

Desired trajectory is defined separately for each subsystem, i.e. platform should move along $\xi_{d}(t)$ and joints of rigid onboard manipulator have to track vector of desired trajectories $q_{r d}(t)$. Moreover, it was assumed that desired trajectories are admissible i.e. that it is possible to move along them without skidding effect in wheels.

\section{Control Algorithms}

\subsection{Mobile Manipulator Moving in Known Terrain - Nonadaptive Case}

Let's consider mobile manipulator with fully known dynamics, given by Eq. 8. If surface, which object is moving on, is known, then reaction forces $F$ are precisely defined. For such case we propose following control law

$$
\begin{gathered}
\left(\begin{array}{c}
u_{m} \\
u_{r}
\end{array}\right)=B_{\xi}^{-1}\left\{J_{\xi}\left(\begin{array}{c}
\ddot{\xi}_{r e f} \\
\ddot{q}_{r e f}
\end{array}\right)+C_{\xi}\left(\begin{array}{c}
\dot{\xi}_{\text {ref }} \\
\dot{q}_{\text {ref }}
\end{array}\right)+\left(\begin{array}{c}
0 \\
D
\end{array}\right)\right. \\
\left.+\left(\begin{array}{c}
F_{\xi} \\
0
\end{array}\right)-K_{d} S\right\}
\end{gathered}
$$

with symbols defined as below

$s=\left(\begin{array}{c}s_{m} \\ s_{r}\end{array}\right)=\left(\begin{array}{c}\dot{\xi} \\ \dot{q}_{r}\end{array}\right)-\left(\begin{array}{c}\dot{\xi}_{r e f} \\ \dot{q}_{r e f}\end{array}\right)$,

where

$$
\begin{aligned}
s_{m} & =\left(\begin{array}{l}
\dot{\xi}_{1}-\dot{\xi}_{1 r e f} \\
\dot{\xi}_{2}-\dot{\xi}_{2 r e f} \\
\dot{\xi}_{3}-\dot{\xi}_{3 r e f}
\end{array}\right)=\left(\begin{array}{l}
\dot{e}_{1}+\Lambda e_{1} \\
\dot{e}_{2}+\Lambda e_{2} \\
\dot{\xi}_{3}-\dot{\xi}_{3 r e f}
\end{array}\right) \\
s_{r} & =\dot{q}_{r}-\dot{q}_{r e f}=\dot{e}_{q}+\Lambda e_{q} \\
e_{1} & =\xi_{1}-\xi_{1 d}, \\
e_{2} & =\xi_{2}-\xi_{2 d}, \\
e_{q} & =q_{r}-q_{r d}, \\
\Lambda & =\Lambda^{T}>0, \quad K_{d}=K_{d}^{T}>0 .
\end{aligned}
$$

It is worth emphasizing that dynamics are calculated for whole mobile object i.e. for SSMP platform and manipulating arm simultaneously. It is a consequence of the fact that dynamic interactions between both subsystems occur and can be really big.

Proof For closed-loop system (8) with feedback control (9), described by equation

$J_{\xi} \dot{s}+C_{\xi} s+K_{d} s=0$,

the following Lyapunov-like function can be chosen

$V\left(\xi, q_{r}\right)=\frac{1}{2} s^{T} J_{\xi} s$ 
Time derivative of $V\left(\xi, q_{r}\right)$ calculated along trajectories of closed-loop system (12) equals to

$\dot{V}=\frac{1}{2} s^{T} \dot{J}_{\xi} s+s^{T} J_{\xi} \dot{s}=-s^{T} K_{d} s \leq 0$.

From La Salle invariance principle it can be concluded that the biggest invariant set equals to $s=0$. Through positive definiteness of matrix $\Lambda$ and definition of sliding variables for platform and onboard manipulator (10)-(11), tracking errors $\left(e_{1}, e_{2}, e_{q}\right) \rightarrow 0$ asymptotically fast.

It is necessary to mention that the third component of $s_{m}$ has to be calculated in different way than other components. Reference trajectory $\xi_{3 r e f}$ must be obtained from condition $u_{3 v}=0$ as a solution of implicit function (14).

\subsection{Expression for Virtual Force - \\ Nonadaptive Case}

Virtual force $u_{3 v}$, obtained from proposed control law (9), has to be equal to zero

$$
\begin{aligned}
u_{3 v}= & -\sin \theta\left[J_{\xi 11} \ddot{\xi}_{1 r e f}+J_{\xi 12} \ddot{\xi}_{2 r e f}+J_{\xi 13} \ddot{\xi}_{3 r e f}\right. \\
& +J_{\xi 14} \ddot{q}_{1 r e f}+J_{\xi 15} \ddot{q}_{2 r e f}+C_{\xi 11} \dot{\xi}_{1 r e f}+C_{\xi 12} \dot{\xi}_{2 r e f} \\
& +C_{\xi 13} \dot{\xi}_{3 r e f}+C_{\xi 14} \dot{q}_{1 r e f}+C_{\xi 15} \dot{q}_{2 r e f}+F_{\xi 1} \\
& \left.-K_{d}\left(\dot{\xi}_{1}-\dot{\xi}_{1 r e f}\right)\right]+\cos \theta\left[J_{\xi 21} \ddot{\xi}_{1 r e f}+J_{\xi 22} \ddot{\xi}_{2 r e f}\right. \\
& +J_{\xi 23} \ddot{\xi}_{3 r e f}+J_{\xi 24} \ddot{q}_{1 r e f}+J_{\xi 25} \ddot{q}_{2 r e f}+C_{\xi 21} \dot{\xi}_{1 r e f} \\
& +C_{\xi 22} \dot{\xi}_{2 r e f}+C_{\xi 23} \dot{\xi}_{3 r e f}+C_{\xi 24} \dot{q}_{1 r e f}+C_{\xi 25} \dot{q}_{2 r e f} \\
& \left.+F_{\xi 2}-K_{d}\left(\dot{\xi}_{2}-\dot{\xi}_{2 r e f}\right)\right] \\
& =0 .
\end{aligned}
$$

Above expression makes possible to get the third reference trajectory $\xi_{3 r e f}$ after integrating $\ddot{\xi}_{3 r e f}$. It is the consequence of the fact that in reality it is impossible to control orientation of the platform because the third actuator (virtual force) does not exist. The remaining elements of sliding vector $s$ can be calculated from Eqs. 10-11 as a linear combination of position and velocity tracking errors of output functions for platform and joints of onboard manipulator.
5.3 Mobile Manipulator Moving in Unknown Terrain - Adaptive Case

Vector of reactive forces and torques is equal to [4]

$$
F_{\xi}=\left(\begin{array}{c}
F_{s} \cos \theta-F_{l} \sin \theta \\
F_{s} \sin \theta+F_{l} \cos \theta \\
M_{r}
\end{array}\right)
$$

with elements defined below

$$
\begin{aligned}
F_{s}= & \sum_{i=1}^{4} F_{s i}=\sum_{i=1}^{4} \mu_{s i} N_{i} \operatorname{sign}\left(v_{x}\right), \\
F_{l}= & \sum_{i=1}^{4} F_{l i}=\sum_{i=1}^{4} \mu_{l i} N_{i} \operatorname{sign}\left(v_{y}\right) \\
M_{r}= & b\left(F_{l 2}+F_{l 3}\right)-a\left(F_{l 1}+F_{l 4}\right) \\
& +c\left(F_{s 3}+F_{s 4}-F_{s 1}-F_{s 2}\right) .
\end{aligned}
$$

Symbols $\mu_{s i}$ and $\mu_{l i}$ denote dry friction coefficients for $i$ th wheel in longitudinal and lateral direction; $N_{i}$ is force acting on $i$ th wheel in vertical direction, depending on weight of the platform; $a$ and $b$ are distances from platform mass center to front and back axis of wheels (we assume $a=b$ ), $c$ is a half of platform width.

More interesting situation holds if some parameters, e.g. interaction forces between wheel and terrain, are uncertain. We assume that form of reaction forces $F_{\xi}$ defined by Eq. 8 is known but friction coefficients $\mu_{s i}$ and $\mu_{l i}$ are constant but unknown. Then reaction forces $F_{\xi}$ given by Eqs. 15-16 can be linearly parameterized in such a way

$F_{\xi}=Y(\dot{\xi}) a^{*}$,

where $Y$ is known regression matrix and $a^{*}$ is a vector of unknown constant real parameters in dynamics.

Table 1 Simulation parameters

\begin{tabular}{llllcl}
\hline Parameter & Value & Unit & Parameter & Value & Unit \\
\hline$m_{p}$ & 60 & $\mathrm{~kg}$ & $\mathrm{c}$ & 0.75 & $\mathrm{~m}$ \\
$m_{k}$ & 3 & $\mathrm{~kg}$ & $I_{p}$ & 10 & $\mathrm{~kg} \cdot \mathrm{m}^{2}$ \\
$R_{w}$ & 0.15 & $\mathrm{~m}$ & $I_{k}$ & 0.034 & $\mathrm{~kg} \cdot \mathrm{m}^{2}$ \\
$l_{1}$ & 0.5 & $\mathrm{~m}$ & $m_{1}$ & 20 & $\mathrm{~kg}$ \\
$l_{2}$ & 1 & $\mathrm{~m}$ & $m_{2}$ & 15 & $\mathrm{~kg}$ \\
$a$ & 0.6 & $\mathrm{~m}$ & $b$ & 0.6 & $\mathrm{~m}$ \\
\hline
\end{tabular}


For mobile manipulator moving in unknown terrain we propose control algorithm given below

$$
\begin{array}{r}
\left(\begin{array}{c}
u_{m} \\
u_{r}
\end{array}\right)=B_{\xi}^{-1}\left\{J_{\xi}\left(\begin{array}{c}
\ddot{\xi}_{r e f} \\
\ddot{q}_{r e f}
\end{array}\right)+C_{\xi}\left(\begin{array}{c}
\dot{\xi}_{\text {ref }} \\
\dot{q}_{\text {ref }}
\end{array}\right)+\left(\begin{array}{c}
0 \\
D
\end{array}\right)\right. \\
\left.+\left(\begin{array}{c}
\hat{F}_{\xi}(t) \\
0
\end{array}\right)-K_{d} s\right\} \\
=B_{\xi}^{-1}\left\{J_{\xi}\left(\begin{array}{c}
\ddot{\xi}_{r e f} \\
\ddot{q}_{r e f}
\end{array}\right)+C_{\xi}\left(\begin{array}{c}
\dot{\xi}_{r e f} \\
\dot{q}_{r e f}
\end{array}\right)+\left(\begin{array}{c}
0 \\
D
\end{array}\right)\right. \\
\left.+\left(\begin{array}{c}
Y \hat{a}(t) \\
0
\end{array}\right)-K_{d} s\right\} .
\end{array}
$$

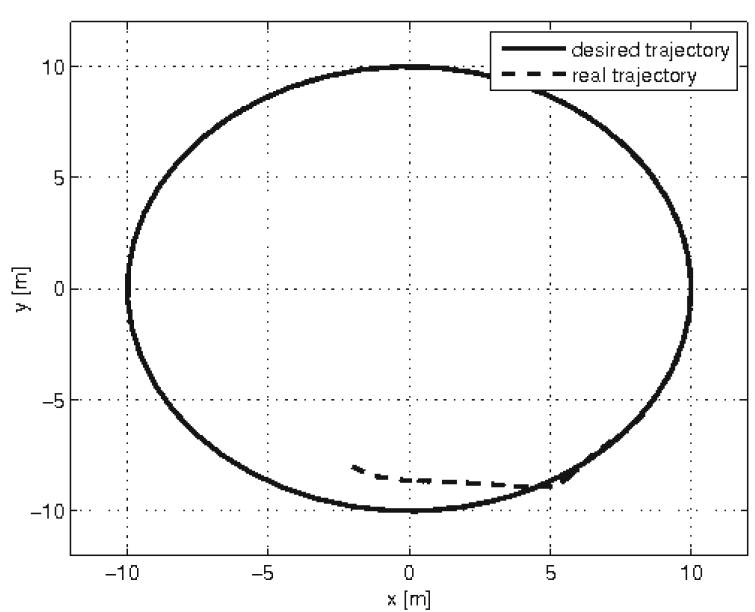

a)

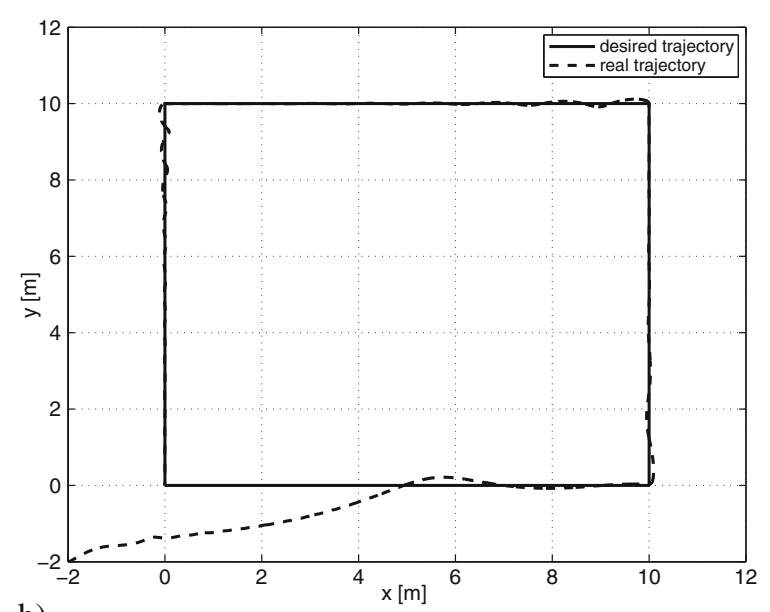

b)

Fig. 2 Trajectory of SSMP platform for known parameters of terrain - nonadaptive case: $\mathbf{a}$ for a circle, $\mathbf{b}$ for a square
In control law (18) estimates $\hat{a}(t)$ of real parameters $a^{*}$ are obtained from adaptation law

$\dot{\hat{a}}=-\Gamma Y^{T} s, \quad \Gamma=\Gamma^{T}>0$,

where $\Gamma$ is the matrix of adaptation gains.

Proof Closed-loop system (8) with feedback control (18) can be presented as

$J_{\xi} \dot{s}+C_{\xi} s+K_{d} s-Y \tilde{a}(t)=0$,

where $\tilde{a}(t)=\hat{a}(t)-a^{*}$ is a parameter error.

For the system (20) the following Lyapunovlike function can be chosen

$V_{a}\left(\xi, q_{r}, \tilde{a}\right)=\frac{1}{2} s^{T} J_{\xi} s+\frac{1}{2} \tilde{a}^{T} \Gamma^{-1} \tilde{a}$.
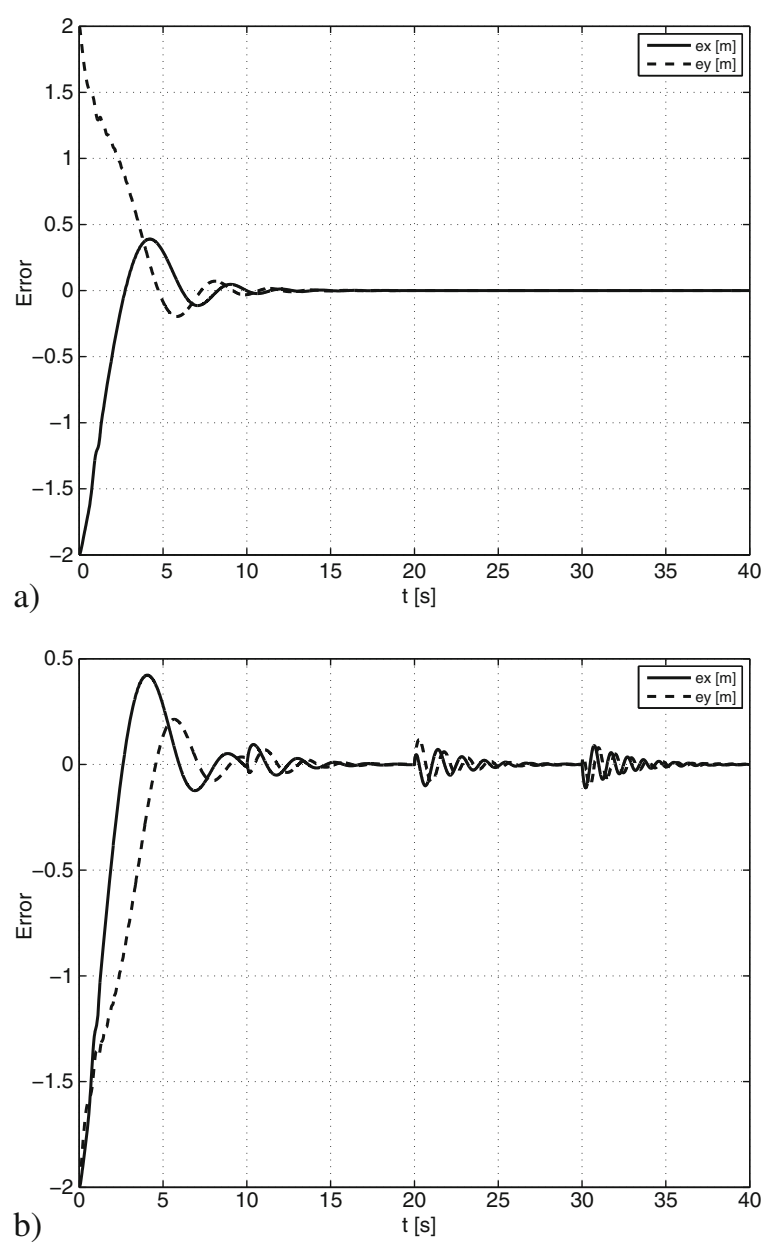

Fig. 3 Position errors of SSMP platform for known parameters of terrain - nonadaptive case: $\mathbf{a}$ for a circle, $\mathbf{b}$ for a square 
Time derivative of $V_{a}\left(\xi, q_{r}, \tilde{a}\right)$ calculated along trajectories of the system (19)-(20) equals to

$\dot{V}_{a}=\frac{1}{2} s^{T} \dot{J}_{\xi} s+s^{T} J_{\xi} \dot{s}+\tilde{a}^{T} \Gamma^{-1} \dot{\tilde{a}}=-s^{T} K_{d} s \leq 0$.

From La Salle-Yoshizawa theorem it can be concluded [5] that the biggest invariant set equals to $s=0$. Moreover, through positive definiteness of matrix $\Lambda$, tracking errors $\left(e_{1}, e_{2}, e_{q}\right)$ go to zero asymptotically fast.
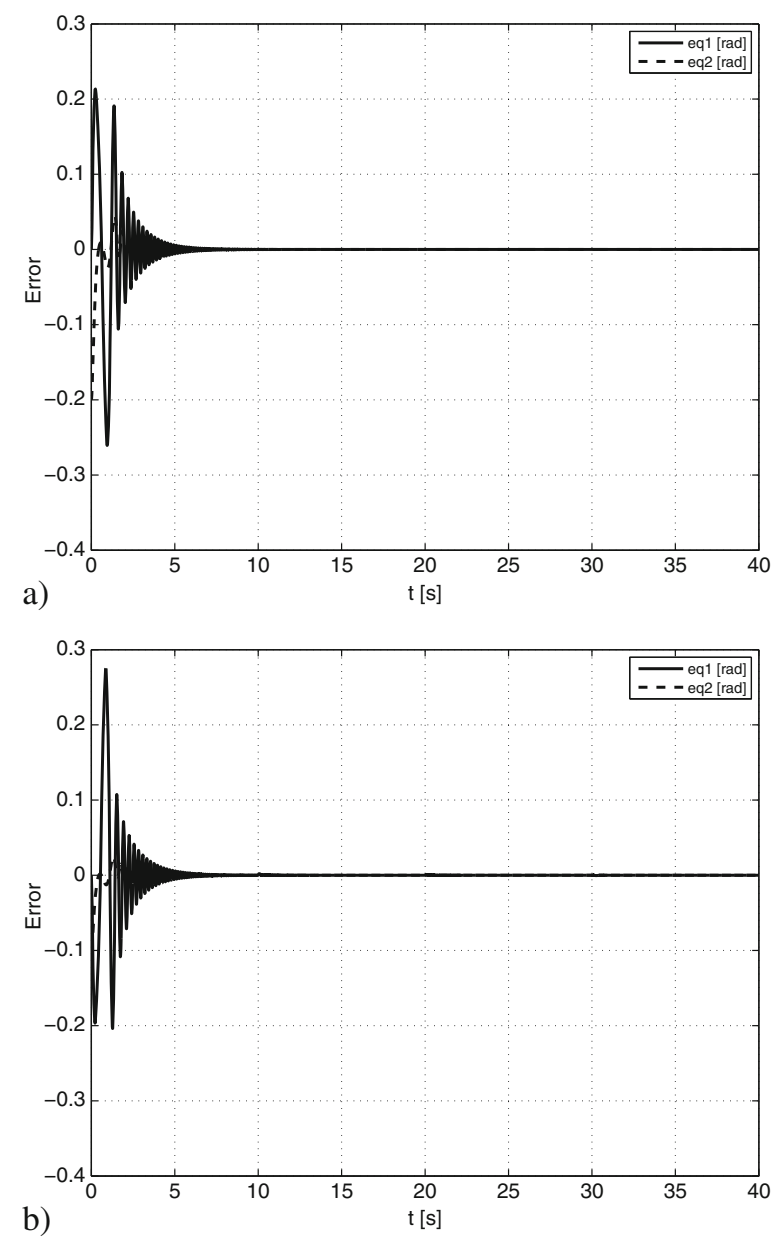

Fig. 4 Joint errors of manipulator for known parameters of terrain - nonadaptive case: $\mathbf{a}$ for a circle, $\mathbf{b}$ for a square

\section{Simulation Results}

The simulations were run with MATLAB package and SIMULINK toolbox. As an object of simulations we have taken SSMP platform equipped with two axes of fixed wheels with $2 \mathrm{R}$ rigid manipulator. The parameters of the platform were: mass of the platform $m_{p}$, mass of the wheel $m_{k}$, platform moment of inertia $I_{p}$ relative $Z_{p}$ axis, wheel moment of inertia $I_{k}$ relative $Z_{p}$ axis, half of platform width $c$, distances $a$ and $b$ from mass center to front and back axis of wheels.
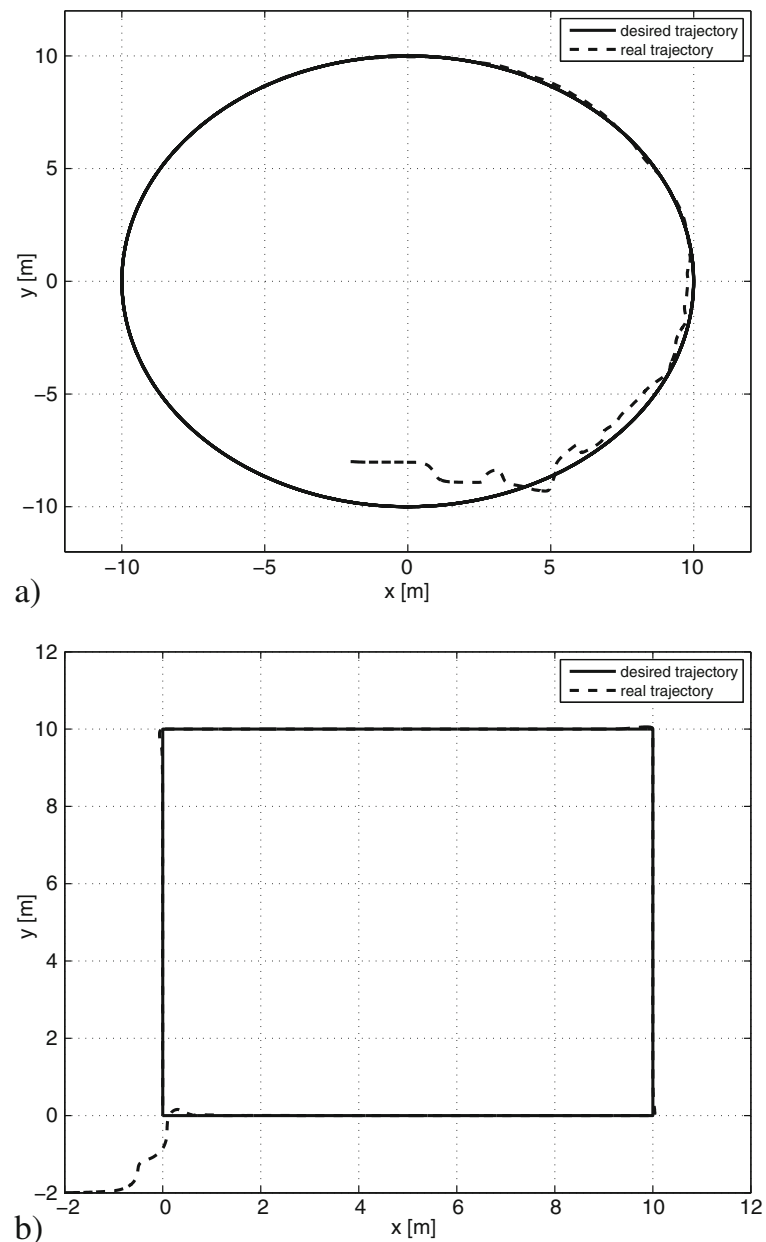

Fig. 5 Trajectory of SSMP platform for unknown parameters of terrain - adaptive case: $\mathbf{a}$ for a circle, $\mathbf{b}$ for a square 
The first link of manipulator was modeled as a cylinder with radius of $R_{w}$, height $l_{1}$ and mass $m_{1}$ and the second link as a bar with length $l_{2}$ and mass $m_{2}$. The parameters of mobile manipulator are presented in Table 1.

As a desired trajectory for the platform two trajectories have been chosen: admissible (circle) and inadmissible (square)

- admissible trajectory - circle with radius $R=$ $10[\mathrm{~m}]$ and frequency $\omega=1[\mathrm{~Hz}]$,

- inadmissible trajectory - square $12[\mathrm{~m}] \times 12[\mathrm{~m}]$, realized in $40 \mathrm{~s}$.
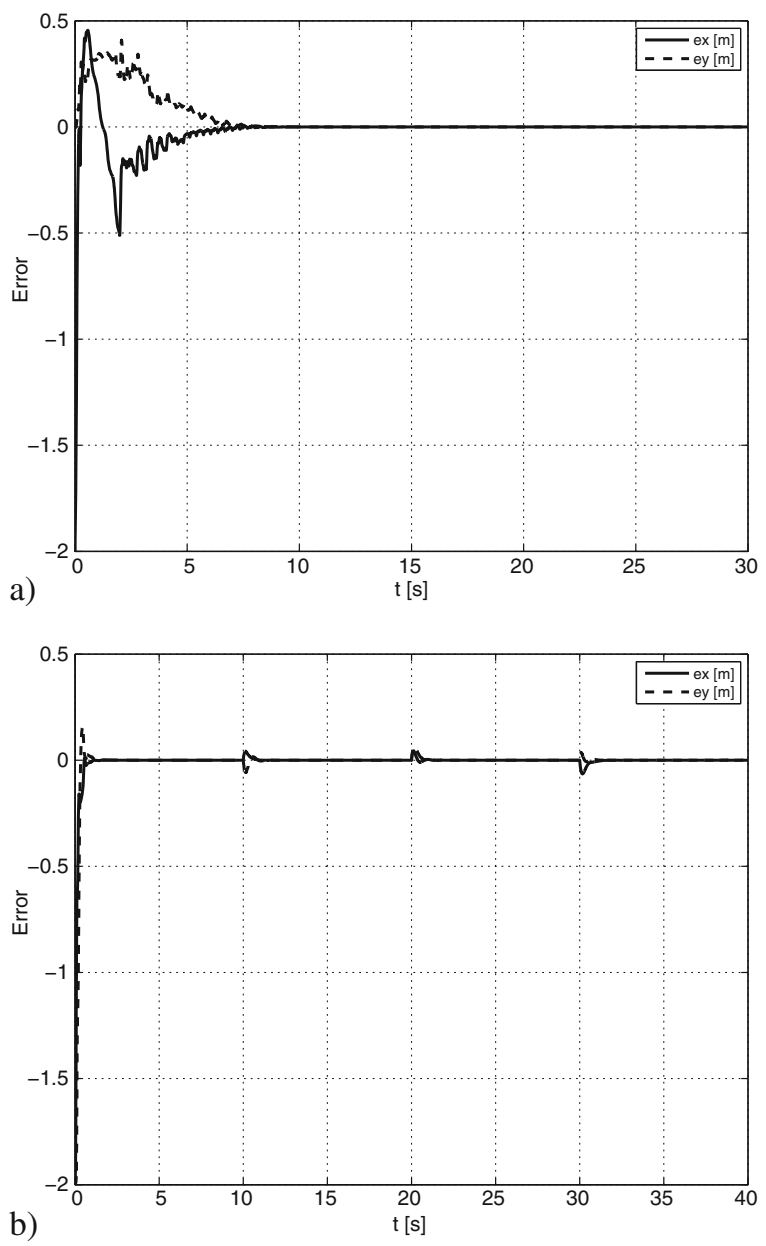

Fig. 6 Position errors of SSMP platform for unknown parameters of terrain - adaptive case: $\mathbf{a}$ for a circle, $\mathbf{b}$ for a square
Desired trajectories for manipulator joints were defined as $q_{1 d}=0$ [rad] and $q_{2 d}=0.1$ [rad].

During simulations, some features of the controlled system has been tested. First, performance of trajectory tracking has been checked for full knowledge about terrain, which platform was moving on. For such a case friction coefficients $\mu_{s i}$ and $\mu_{l i}$ were chosen as equal to 0.5 and they were known. Next, the same simulations have been made for adaptive case - for unknown ground parameters: $\mu_{s i}=0.9$ and $\mu_{l i}=0.1$.
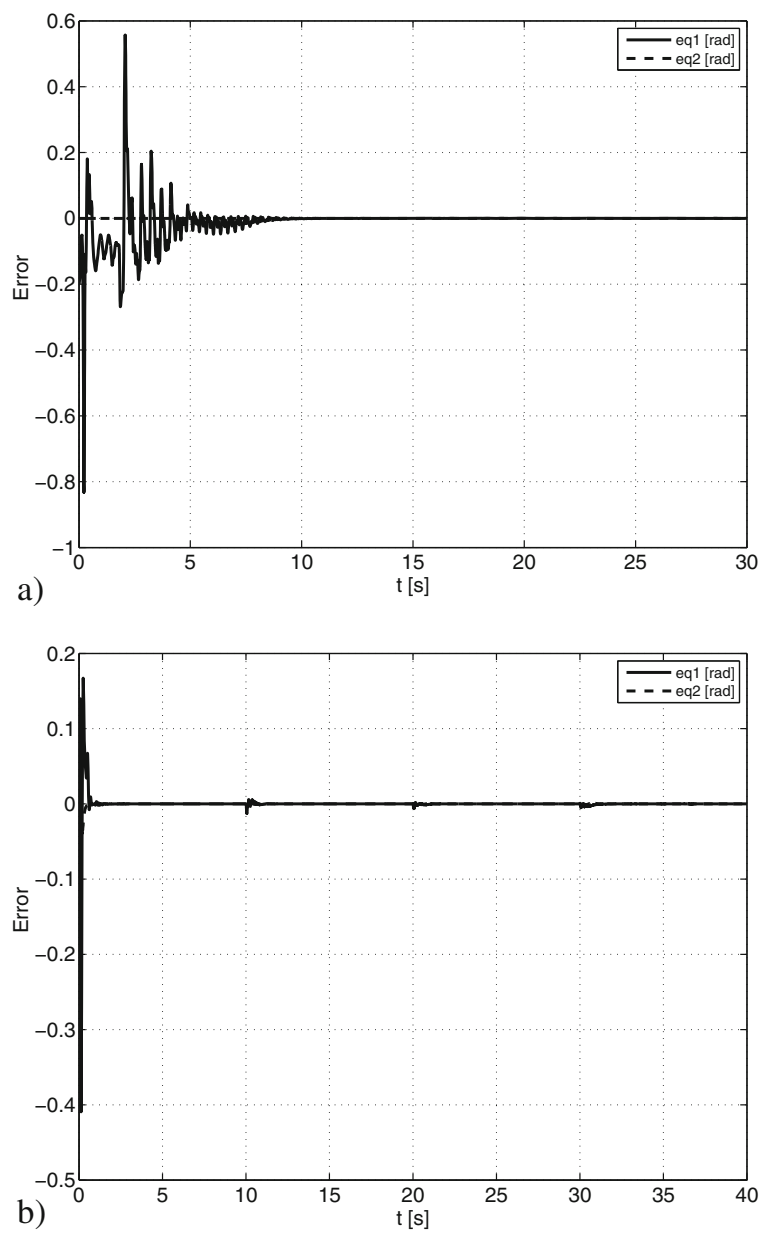

Fig. 7 Joint errors of manipulator for unknown parameters of terrain - adaptive case: $\mathbf{a}$ for a circle, $\mathbf{b}$ for a square 


\subsection{Robot Motion in Known Environment}

Figs. 2, 3, 4 have been made for a case of known ground parameters - for nonadaptive case. Tracking of the desired trajectory for the mobile platform has been presented in Figs. 2-3 whereas in Fig. 4 position errors in manipulator joints have been plotted. Regulation parameters were chosen as $K_{d}=50$ and $\Lambda=10$.

\subsection{Robot Motion in Unknown Environment}

Figs. 5, 6, 7 have been made for a case of unknown ground parameters, i.e. for adaptive case. Tracking of the desired trajectory for the mobile platform has been presented in Figs. 5-6 and in
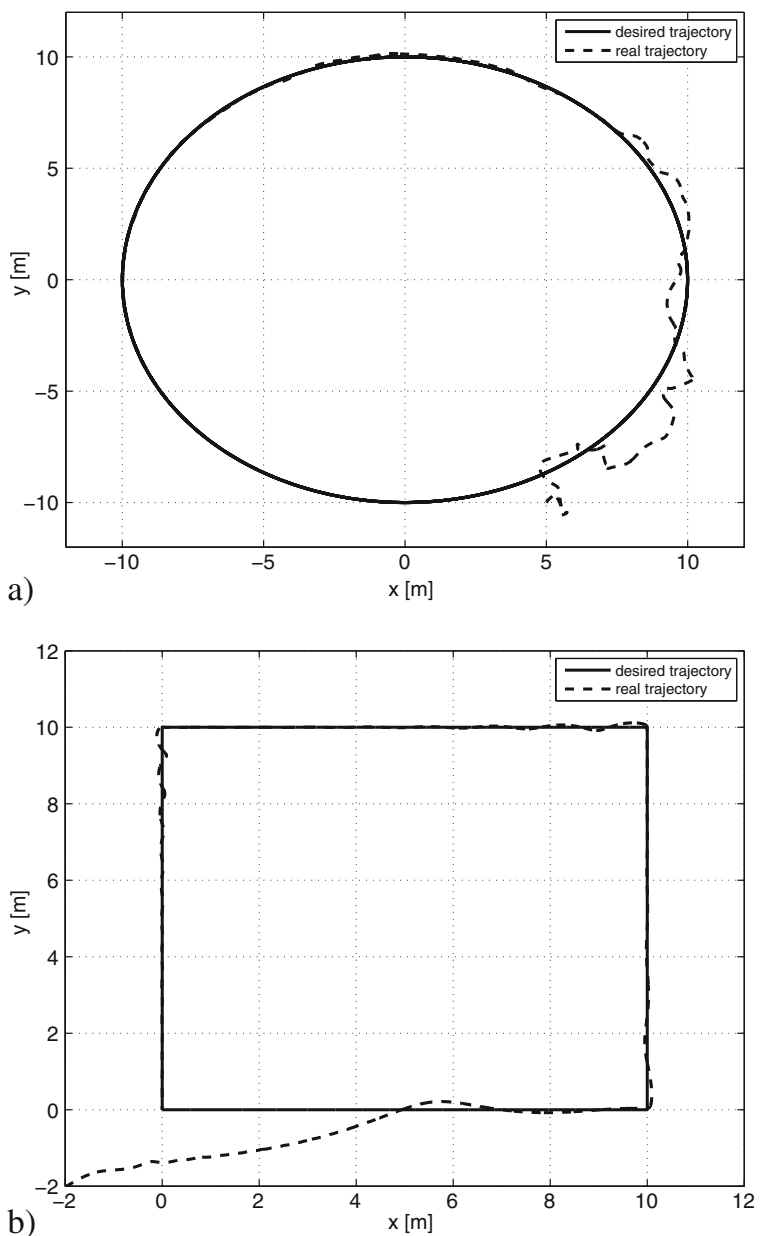

Fig. 8 Trajectory of SSMP platform for changing parameters of terrain - adaptive case: $\mathbf{a}$ for a circle, $\mathbf{b}$ for a square
Fig. 7 position errors in manipulator joints have been plotted. Regulation parameters were chosen as $K_{d}=50, \Lambda=10$ and $\Gamma=10$ for a circle and $K_{d}=500, \Lambda=10$ and $\Gamma=10$ for a square trajectory.

\subsection{Robot Motion in Changing Environment}

Simulation has been made also for changing parameters of ground. First, friction coefficients have been selected in simulated environment as $\mu_{s i}=$ 0.1 and $\mu_{l i}=0.9$ for time interval $[0,10] \mathrm{s}$. Next, after $10 \mathrm{~s}$ parameters changed rapidly on values $\mu_{s i}=0.4$ and $\mu_{l i}=0.6$. Nevertheless, trajectory tracking has been realized properly, what can be seen in Fig. 8. Tracking for the mobile platform
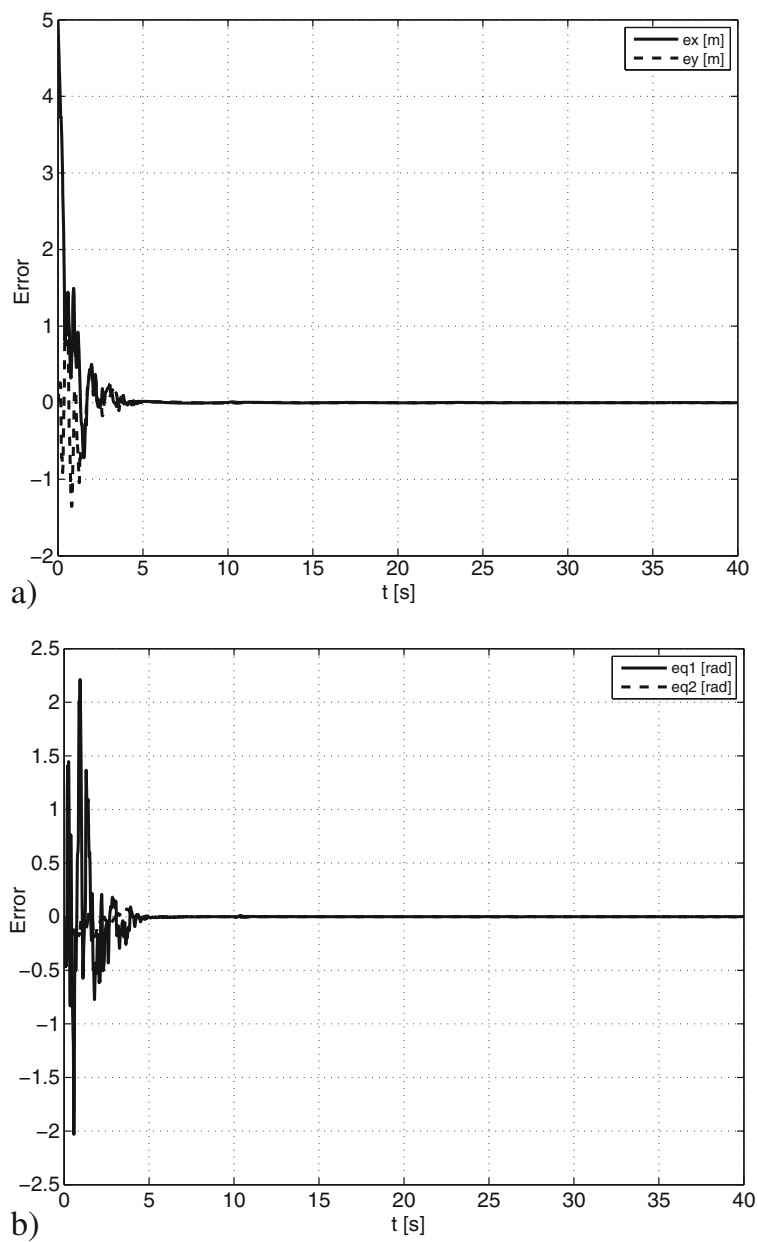

Fig. 9 Position errors for changing parameters of terrain: a errors for SSMP platform, $\mathbf{b}$ joint errors for manipulator 

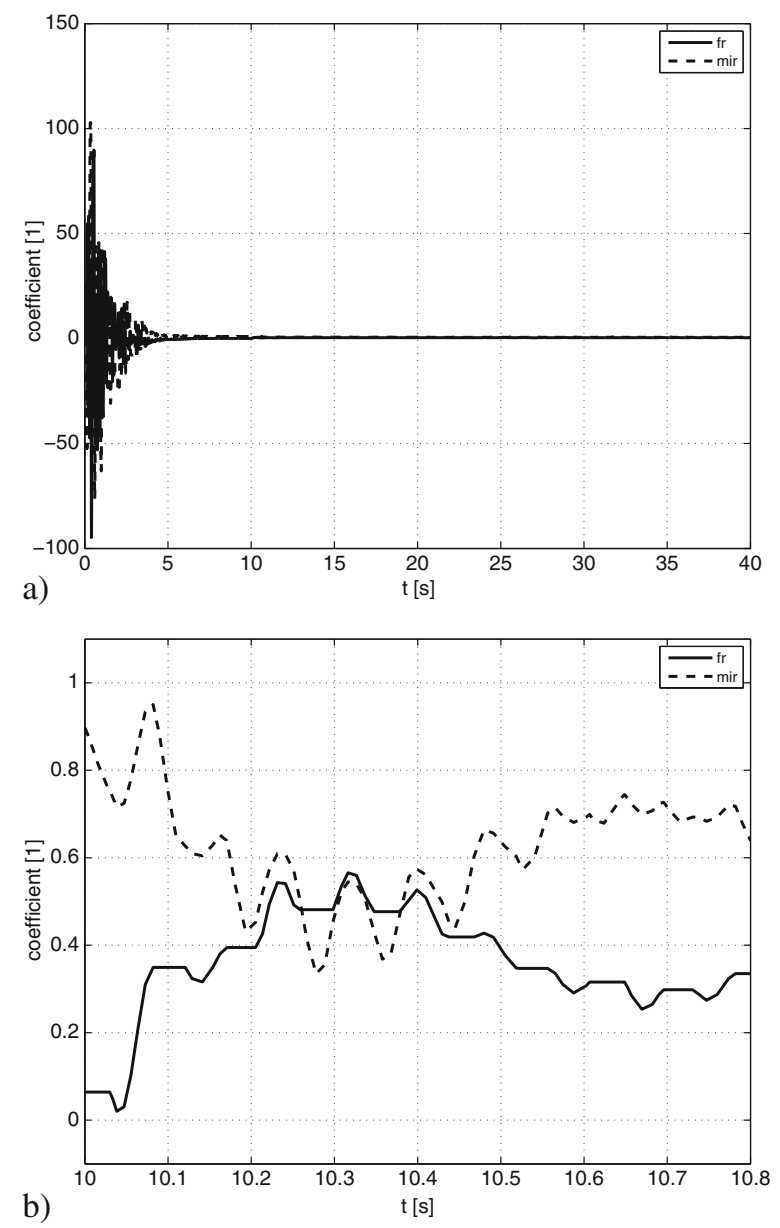

Fig. 10 Estimates of terrain parameters: a in $40 \mathrm{~s}$, b zoom of plot after changing parameters

has been presented in Figs. 8 and 9a whereas in Fig. $9 \mathrm{~b}$ position errors in joints of manipulator have been plotted. In Fig. 10 estimates of terrain parameters have been presented.

\section{Conclusions}

In this paper new approach to control mobile manipulator with skid-steering mobile platform has been presented and discussed. Instead of artificial assumption on object velocity, concept of virtual force has been proposed. Such description allows to solve control problem for system with deficit of input signals. Numerous simulations have confirmed, that such concept can find practical applications for nonholonomic mobile manipulators moving with slippage effect, even if the terrain, which platform is moving on, is unknown and changes during motion.

Open Access This article is distributed under the terms of the Creative Commons Attribution License which permits any use, distribution, and reproduction in any medium, provided the original author(s) and the source are credited.

\section{References}

1. Baillieul, J.: Kinematic programming alternatives for redundant manipulators. In: Proceedings of the IEEE International Conference on Robotics and Automation, pp. 722-728. St. Louis (1985)

2. Caracciolo, L., De Luca, A., Iannitti, S.: Trajectory tracking control of a four-wheel differentially driven mobile robot. In: Proc. of the IEEE Int. Conf. on Robotics and Automation, pp. 2632-2638. Detroit, Michigan (1999)

3. D'Andrea-Novel, B., Campion, G., Bastin, G.: Control of wheeled mobile robots not satisfying ideal velocity constraints: a singular perturbation approach. Int. J. Robust. Nonlin. Control. 5(4), 243-267 (1995)

4. Kozłowski, K., Pazderski, D.: Modeling and control of a 4-wheel skid-steering mobile robot. Int. J. Appl. Math. Comput. Sci. 14(4), 4 (2004)

5. Krstić, M., Kanellakopoulos, I., Kokotović, P.: Nonlinear and Adaptive Control Design. John Wiley and Sons, New York (1995)

6. Mazur, A., Kȩdzierski, J.: Nonlinear control law for nonholonomic balancing robot. In: Arreguin, J. (ed.) ITECH Automation and Robotics, pp. 87-96. Academic Press, Vienna (2008)

7. Canudas de Wit, C., Siciliano, B., Bastin, G.: Theory of Robot Control. Springer-Verlag, London (1996)

8. Mohammadpour, E., Naraghi, M., Gudarzi, M.: Posture stabilization of skid steer wheeled mobile robots. In: Proceedings of the IEEE International Conference on Robotics Automation and Mechatronics, pp. 163169. Singapore (2010)

9. Pazderski D, Kozłowski K (2008) Trajectory tracking of underactuated skid-steering robot. In: Proc. of the American Control Conference, Washington, DC, pp 3506-3511

10. Tchoń K, Jakubiak J, Zadarnowska K (2005) An extended jacobian inverse kinematics algorithm for doubly nonholonomic mobile manipulators. In: Proc. of the IEEE Int. Conf. on Robotics and Automation, Barcelona, pp 1548-1553 